Michel Habib Fabrice Robichon

\section{ADRESSE}

M. Habib : neurologue des hôpitaux. F. Robichon: attaché de recherches. Clinique de neurologie, CHU Timone, boulevard Jean-Moulin, 13385 Marseille Cedex 5, France.

\title{
Les mécanismes cérébraux de la lecture : un modèle en neurologie cognitive
}

L'étude anatomo-clinique des alexies, pertes de la lecture consécutives à des lésions focales du cerveau, a donné naissance aux conceptions classiques de déconnexion inter- ou intrahémisphérique des syndromes neuropsychologiques. La révolution cognitiviste, au cours des vingt dernières années, également fondée sur les dissociations observées lors de perturbations acquises de la lecture, a permis de distinguer les dyslexies profondes (phonologiques) des dyslexies de surface (lexico-sémantiques). Actuellement, les méthodes d'imagerie fonctionnelle du cerveau appliquées à la lecture laissent entrevoir un rapprochement des conceptions anatomo-clinique et cognitiviste. Enfin, l'étude du développement s'impose de plus en plus pour préciser les fonctions cognitives. C'est dans le domaine de la lecture, avec les dyslexies liées au développement, que se réalisent actuellement les premiers travaux permettant de mettre en relation des déficits de processus cognitifs précis avec la mise en place défectueuse de certains circuits cérébraux.

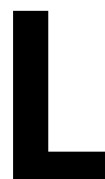

'histoire des relations entre le cerveau et le fonctionnement cognitif humain a connu, tout au long du siècle qui se termine, trois périodes ayant chacune apporté sa contribution à l'état actuel de nos connaissances: la période anatomoclinique, qui a débuté à la fin du siècle dernier, la période cognitiviste, née au début des années 1970, et la période actuelle, qui, grâce à l'apport des moyens modernes d'imagerie fonctionnelle du cerveau, tente, encore difficilement, d'établir le lien entre les deux approches précédentes, une démarche que l'on qualifie volontiers actuellement de «neurologie cognitive». Tout au long de cette lente évolution des idées, le domaine de la lecture a occupé et occupe encore, par son exemplarité, une place privilégiée, sans doute en raison de la situation particulière du langage écrit, à l'interface entre vision et langage, deux des aspects les plus directement et objectivement explorables des fonctions du cerveau humain.

Le propos du présent article est 
d'illustrer, à l'aide de l'exemple de la lecture, et à la lumière d'un panorama historique de la progression des connaissances, le mode de raisonnement de la neurologie cognitive et de proposer quelques réflexions qui pourraient être utiles pour orienter les recherches dans les années à venir.

\section{L'alexie et les théories déconnexionnistes}

L'alexie, perte spécifique des facultés de décodage du langage écrit consécutive à une lésion acquise du cerveau, a longtemps constitué le fondement de tous les raisonnements quant au substrat cérébral de la lecture. Le premier cas d'alexie publié par Dejerine [1] en 1891 était celui d'un sujet ayant présenté, à la suite d'une petite lésion, «de la taille d'une pièce de 5 francs", située à la jonction temporo-occipitale de l'hémisphère gauche (précisément la région dénommée pli courbe ou gyrus angulaire), une perte totale de la faculté de comprendre le langage écrit associée à une perte de l'expression écrite. Ce syndrome est connu depuis lors sous le nom d'«alexie-agraphie de Dejerine». Cette constatation conduisit son auteur à élaborer une première ébauche d'un modèle anatomo-fonctionnel de la lecture, selon lequel il existerait dans cette petite zone de cortex, située, dans l'hémisphère gauche du langage, à égale distance des zones proprement langagières et des zones visuelles, un «centre» qui contiendrait, selon ses propres termes, «la mémoire optique des lettres", de sorte que sa destruction pathologique serait responsable à la fois d'une perte de la compréhension et de l'expression du langage écrit. L'année suivante, le même Dejerine publia une observation plus complexe [2] qui devait à la fois asseoir et compléter sa théorie de la mémoire optique des mots. Le sujet, également à la suite d'un accident vasculaire cérébral, était lui aussi devenu alexique, mais, de manière surprenante, pouvait encore parfaitement écrire, sans, toutefois, pouvoir relire ce qu'il venait d'écrire. Il était cependant encore capable de reconnaître les mots épelés oralement et épeler lui-même des mots. En outre, il présentait un déficit de la vision dans la moitié droite du champ visuel (hémianopsie droite). Ce syndrome, depuis lors fréquemment observé en neurologie clinique, porte le nom d' «alexie sans agraphie ou alexie pure», ou encore, comme le dénommait Dejerine "cécité verbale pure». Le raisonnement de Dejerine était que le «centre de l'image des lettres » devait être resté intact (puisqu'il était encore capable de produire du langage écrit) mais qu'il devait être privé de toute information visuelle: en d'autres termes, le gyrus angulaire devait être privé d'afférences visuelles. Quatre ans plus tard, peu de temps avant sa mort, le même patient fut victime d'un nouvel incident cérébral qui le priva cette fois de la capacité d'écrire, transformant le tableau d'alexie sans agraphie en une alexie avec agraphie, similaire à celle du patient de 1891. Dejerine fit alors l'hypothèse que ce deuxième accident devait avoir cette fois bel et bien détruit le gyrus angulaire, à l'instar de l'observation précédente. Les constatations autopsiques confirmèrent tout à fait cette prédiction en montrant deux lésions d'âges différents, l'une récente détruisant électivement le gyrus angulaire gauche, l'autre plus ancienne (donc correspondant au premier accident vasculaire) intéressant à la fois le cortex occipital interne gauche et la partie la plus ventrale du splénium du corps calleux.

Dans son mémoire célèbre sur les syndromes de déconnexion, mémoire qui a profondément marqué le raisonnement neurologique de ces trente dernières années, Geschwind [3] regardait l'alexie pure comme le modèle par excellence de déconnexion interhémisphérique. Se fondant sur une observation [4] de lésion occipitale gauche ayant détruit, comme dans celle de Dejerine, à la fois le cortex visuel et le splenium du corps calleux, Geschwind postulait que l'hémianopsie droite, conséquence de la lésion du système visuel gauche, privait l'hémisphère gauche de toute afférence visuelle directe, de sorte que l'information sur le mot lu, arrivée exclusivement à l'hémisphère droit, devait nécessairement, pour parvenir au gyrus angulaire gauche chargé de son identification, emprunter les fibres calleuses 12. Geschwind N. Specializations of the
human brain. Sci Am 1979; 241: 158-68. 
(figure 1A). Ces dernières étant interrompues par la lésion du splénium, le mot ne pouvait donc plus être identifié. Diverses études de séries de patients ayant présenté ce type de lésion, dont la cause la plus fréquente est l'infarctus du territoire de l'artère cérébrale postérieure gauche (figure 1B), montrent que la majorité d'entre eux ont des troubles de la lecture [5]. Qui plus est, il a été démontré [6], en comparant des cas d'infarctus de ce territoire avec et sans alexie, que le point commun des cas sans alexie était l'absence de lésion du splenium, confirmant le rôle de l'atteinte spléniale dans le mécanisme de l'alexie. Toutefois, cette interprétation a été à maintes reprises contestée, en particulier au vu d'observations d'alexie pure sans hémianopsie [7, 8], l'absence d'hémianopsie rendant de fait beaucoup plus fragile une explication en terme de déconnexion interhémisphérique.

Une contribution déterminante à ce débat fut celle de Damasio [9, 10]. Ce dernier remarqua que, dans son travail de 1892, Dejerine avait décrit «l'hémianopsie» de son patient comme incomplète, caractérisée surtout par un trouble de la vision des couleurs dans l'hémichamp visuel droit. En fait, ce que Dejerine appelait hémianopsie était seulement ce que l'on dénomme aujourd'hui une hémiachromatopsie: donc, le patient n'était pas hémianopsique et, contrairement à l'interprétation qui en avait toujours été fournie, l'observation de Dejerine n'était pas, à proprement parler, un cas de déconnexion interhémisphérique. Du reste, Damasio soulignait que Dejerine lui-même ne faisait pas intervenir, dans son interprétation, la lésion calleuse, mais considérait plutôt, comme il le précisa plus tard [11], que la région cruciale était une petite zone de substance blanche, à proximité du ventricule latéral, capable de détruire à la fois les fibres unissant le cortex visuel gauche au gyrus angulaire et celles unissant les deux régions visuelles, droite et gauche (figure 1C). En définitive, on admet actuellement que le syndrome d'alexie pure de Dejerine est bel et bien un syndrome de déconnexion entre le système visuel et les aires du langage, mais dont le mécanisme peut être soit intrahémisphérique, comme dans le cas de Dejerine, soit interhémisphérique, comme dans le cas de Geschwind et Fusillo [4].

\section{Le modèle de Dejerine/Geschwind et ses faiblesses}

Si l'on s'en tient ainsi aux données issues des études anatomocliniques, on peut imaginer les mécanismes cérébraux menant de la perception d'un mot écrit à sa production orale selon le modèle schématisé sur la figure 2. Ce modèle, proposé par Geschwind [3,12] conçoit plusieurs étapes successives: l'information écrite arrive en premier lieu au niveau des aires visuelles primaires d'où elle converge vers les aires associatives visuelles de l'hémisphère gauche. De là, elle parvient au gyrus angulaire gauche, tout proche, dont la situation de plaque tournante entre le système visuel et les aires du langage en fait le candidat idéal pour établir la correspondance entre l'image du mot et son codage phonologique. L'importance qu'accordait Geschwind au gyrus angulaire était en partie empirique, fondée sur l'observation des troubles liés à sa destruction, mais aussi et surtout intuitive, en raison de sa nature de cortex multimodal, véritable zone de transition entre les cortex associatifs spécifiques des modalités auditive et

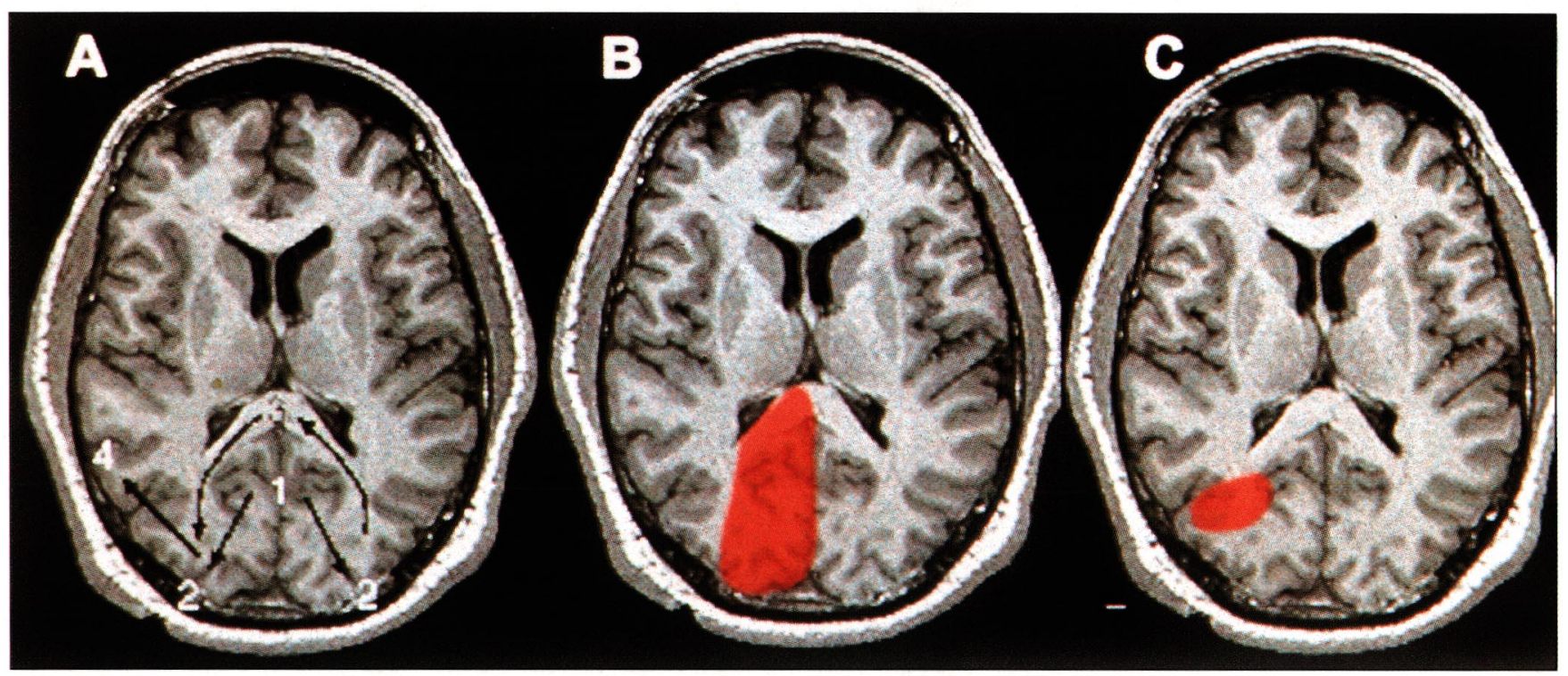

Figure 1. Siège des lésions provoquant une alexie pure. A. trajet des fibres visuelles. 1: cortex visuel primaire; 2: aires visuelles associatives ; 3 : splénium du corps calleux (fibres interoccipitales) ; 4 : gyrus angulaire. B. lésion occipitale gauche étendue [4]: la lésion détruit les voies et aires visuelles, provoquant une hémianopsie droite totale et le splénium du corps calleux, interrompant le passage des informations visuelles de l'hémisphère droit vers le gyrus angulaire gauche (déconnexion interhémisphérique). C. lésion de la substance blanche profonde [2], déconnectant à la fois les fibres calleuses et les fibres occipito-pariétales gauches (déconnexion intrahémisphérique). 


\section{RÉFÉRENCES}

13. Marshall JC, Newcombe F. Patterns of paralexia: a psycholinguistic approach. $J$ Psycholinguist Res 1973; 2 : 175-99.

14. Beauvois MF, Derouesné J. Phonological alexia. Three dissociations. J Neurol Neurosurg Psychiatr 1979; 42: 1115-24.

15. Ellis AW, Young AW. Human cognitive neuropsychology. Hillsdale, New Jersey: Elrbaum Associates, 1987.

16. Landis T, Regard M, Serrat A. Iconic reading in a case of alexia without agraphia caused by a brain tumor : a tachistoscopic study. Brain Lang 1980; 11 : 45-53.

17. Shallice T, Saffran E. Lexical processing in the absence of explicit word identification : evidence from a letter-by-letter reader. Cogn Neuropsychol 1986; 3: 429-59.

18. Coslett HB, Saffran EM, Greenbaum S, Schwartz H. Reading in pure alexia. The effect of strategy. Brain 1993; 116: 21-37.

19. Démonet JF, Wise R, Frackowiak RSJ. Les fonctions linguistiques explorées en tomographie par émission de positons. médecine/sciences 1993; 9: 934-42.

20. Sergent J. Brain-imaging studies of cognitive functions. Trends Neurol Sci 1994; 17: $221-7$

21. Habib M, Démonet JF. Cognitive neuroanatomy of language: the contribution of functional neuroimaging. Aphasiology 1996 (sous presse).

22. Sergent J. De la musique au cerveau par l'intermédiaire de Maurice Ravel. médecine/sciences $1993 ; 9$ : 50-8.

23. Petersen SE, Fox PT, Snyder AZ, Raichle ME. Activation of extrastriate and frontal cortical areas by visual words and word-like stimuli. Science 1990 ; 249 : 1041-4.

24. Howard D, Patterson K, Wise R, Brown WD, Friston K, Weiller C, Frackowiak R. The cortical localisation of the lexicons : PET evidence. Brain 1992; 115 : 1769-82.

25. Price CJ, Wise RSJ, Watson JDG, Patterson K, Howard D, Frackowiak RSJ. Brain activity during reading. The effects of exposure duration and task. Brain 1994; 117: 1255-69.

26. Castles A, Coltheart M. Varieties of developmental dyslexia. Cognition 1993; 47 :

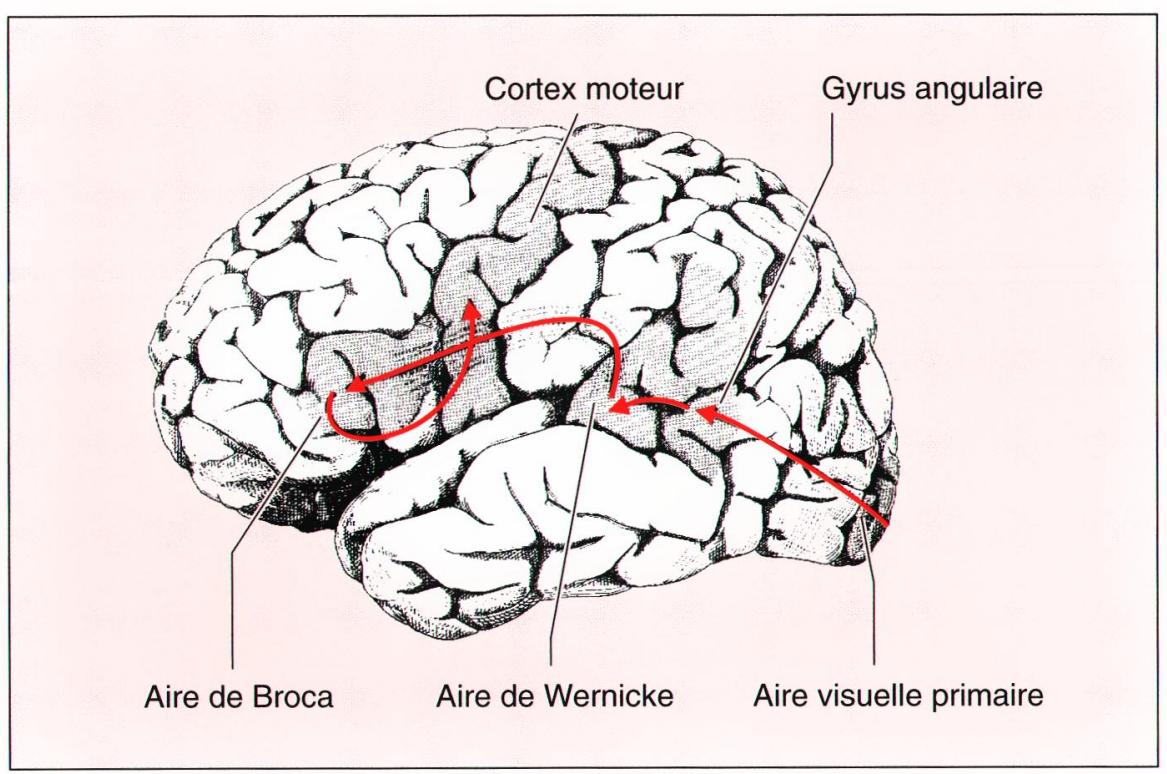

Figure 2. Le modèle classique de la lecture. L'information écrite arrive en premier lieu au niveau des aires visuelles primaires d'où elle converge vers les aires associatives visuelles de l'hémisphère gauche. De là, elle parviendrait au gyrus angulaire gauche, plaque tournante entre les informations visuelles et les aires du langage. L'étape suivante se ferait dans l'aire de Wernicke, où s'établiraient les liens entre mot et concept sémantique. Le mot ayant subi un recodage phonologique et acquis un sens, serait transféré à l'aire de Broca qui programme les actes moteurs nécessaires à sa prononciation [3, 12].

visuelle. En d'autres termes, Geschwind présumait qu'à ce niveau s'établissait une «transformation du mot écrit en sa forme sonore ", transformation nécessaire au passage aux étapes suivantes. L'étape juste ultérieure ferait intervenir le cortex temporal postéro-supérieur, c'est-à-dire l'aire de Wernicke, au niveau de laquelle on pensait généralement que s'établissaient les liens entre le mot et les concepts qui lui sont sémantiquement rattachés. Enfin, le mot ayant subi un recodage phonologique et acquis un sens, serait transféré, grâce au "faisceau arqué » reliant l'aire de Wernicke à l'aire de Broca, à cette dernière qui programmerait les actes moteurs nécessaires à sa prononciation. Ce modèle, encore largement enseigné aujourd'hui, a subi de sérieuses attaques et s'avère actuellement invalide sur plusieurs points.

\section{Deux voies valent mieux qu'une : l'apport de la neuropsychologie cognitive}

La première de ces contestations est venue du puissant courant cognitiviste introduit dans les années 1970 (et dont l'influence est encore considérable), qui considère que la lecture peut se faire selon deux procédures distinctes, l'une, dite de surface, opérant une conversion des graphèmes (caractères élémentaires du langage écrit) en phonèmes (leurs correspondances sonores), et une seconde, dite profonde, capable de lire les mots de manière globale en les adressant à un lexique donnant directement accès à leur sens. La procédure de surface est appelée voie phonologique et la procédure profonde, voie sémantique (ou lexico-sémantique). C'est 


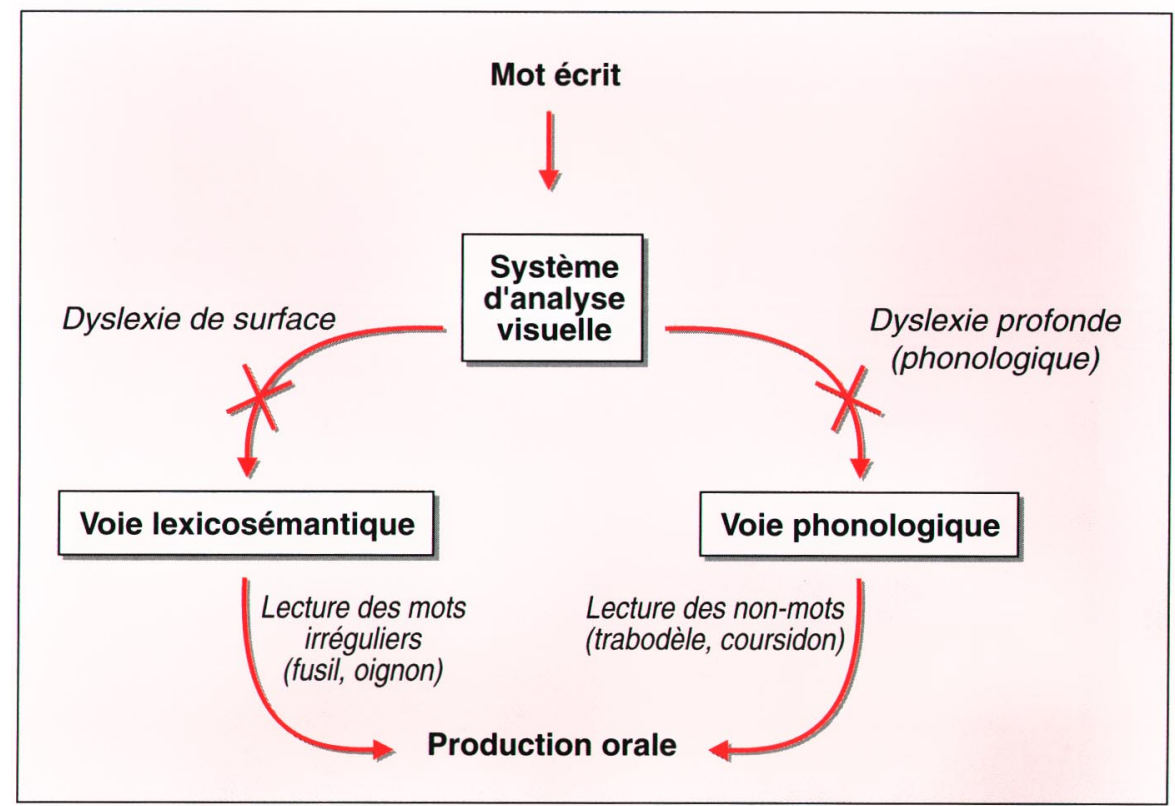

Figure 3. Les deux voies de la lecture. D'après les théories cognitivistes, le mot écrit peut être traité selon deux procédures différentes, dont l'atteinte spécifique donne lieu à deux types de dyslexies (profonde et de surface). La lecture de mots irréguliers ne peut se faire que par la voie sémantique, celle de non-mots que par la voie phonologique.

l'application de ce modèle à deux voies (figure 3) à l'étude des alexies qui a fait entrer la psychologie cognitive dans le champ de la neuropsychologie [13], avec l'individualisation, sur la base de leurs erreurs en lecture de mots isolés, de deux types de patients alexiques: ceux chez lesquels le trouble de la lecture intéresse spécifiquement les mots sans signification (tels que «traboru» ou «sigrodelle»), qui ne peuvent être lus que par la procédure de surface, alors que la lecture des mots réels est nettement mieux préservée (bien que non intacte), et ceux présentant la dissociation inverse, c'est-à-dire que les mots sans signification ne leur posent aucun problème alors que les mots irréguliers (comme «femme» ou «fusil»), qui ne peuvent être lus convenablement que par adressage lexical, donnent lieu à des erreurs dites de "régularisation» («fème» ou «fusile»). Depuis lors, on admet que les patients du premier type ont une "dyslexie profonde» (ou une «alexie phonologique» [14]) et ceux du deuxième type une «dyslexie de surface»*. Ainsi s'ouvrit une nouvelle ère dans l'étude des patients alexiques, où le raisonnement ne portait plus sur les lésions et la fonction perdue par les structures cérébrales détruites, mais sur les mécanismes sous-jacents aux erreurs analysées. Ainsi, plus récemment, ont été individualisés des cas d'alexie où le trouble semblait siéger en amont de la divergence en deux voies, c'est-àdire au niveau des étapes précoces du traitement visuel des mots, étapes souvent rapportées au fonctionnement d'un module d'analyse visuelle des lettres et des mots [15] où seraient extraites, en parallèle, les différentes caractéristiques physiques et spatiales des traits composant les lettres et les modalités d'organisation de ces dernières pour constituer le riques»). La forme la plus fréquente de ces dyslexies périphériques est sans doute celle dite dyslexie «lettrepar-lettre» encore appelée dyslexie par épellation, qui n'est autre que la forme évolutive la plus fréquente de l'alexie pure de Dejerine, mais envisagée non plus sous l'angle neurolo-

\footnotetext{
* Le terme de «dyslexie» ne réfere pas aux dyslexies de développement de l'enfant dont il sera question plus bas, mais est employé ici comme synonyme d'alexie, terme auquel il est généralement préféré par les psychologues cognitivistes et qui est entré depuis lors sous cette acception dans la littérature neurologique.
} mot lu (dyslexies dites «périphé- gique mais sous celui des mécanismes cognitifs. Un aspect surprenant du comportement de ces patients est leur capacité à identifier certains mots de manière globale et non consciente [16-18]: en effet, en condition de lecture normale, ils ne peuvent lire des mots même simples que laborieusement, en dénommant chaque lettre, ce qui nécessite un temps considérable. Lorsqu'on leur présente les mêmes mots, mais projetés très brièvement sur le centre d'un écran, de telle sorte qu'ils ne puissent pas tenter d'utiliser leur stratégie de lecture lettre par lettre, ils sont toujours incapables de les dénommer mais on peut parfois mettre en évidence, à leur insu, des capacités non conscientes de traitement lexical et sémantique du mot. Ce phénomène est volontiers interprété comme l'utilisation, à un niveau infra-conscient, de capacités de l'hémisphère droit, capacités qui pourraient être inhibées par l'utilisation excessive de la stratégie d'épellation, qui s'appuie, elle, totalement sur l'hémisphère gauche lésé.

D'une manière générale, il faut bien admettre que l'approche cognitiviste, si elle a permis de remettre en cause certains dogmes probablement erronés dans les conceptions classiques de Dejerine et Geschwind, n'a pas fait réellement progresser nos connaissances des mécanismes cérébraux eux-mêmes: en particulier, l'étude des lésions responsables de dyslexie phonologique et de surface n'a pas permis de distinguer clairement les zones corticales sous-jacentes aux deux mécanismes postulés par les théories cognitivistes (figure 4).

\section{Apport de l'imagerie fonctionnelle}

Depuis quelques années, à l'approche anatomo-clinique classique et à celle de la neuropsychologie cognitive est venue s'ajouter une troisième source d'information sur les liens entre cerveau et cognition, la neuroanatomie fonctionnelle qui étudie in vivo le cerveau normal à l'aide de méthodes d'imagerie fonctionnelle chez des sujets engagés dans une tâche cognitive choisie en fonction du domaine étudié (méthode d' "activation cognitive»). Le développement considérable de ces 


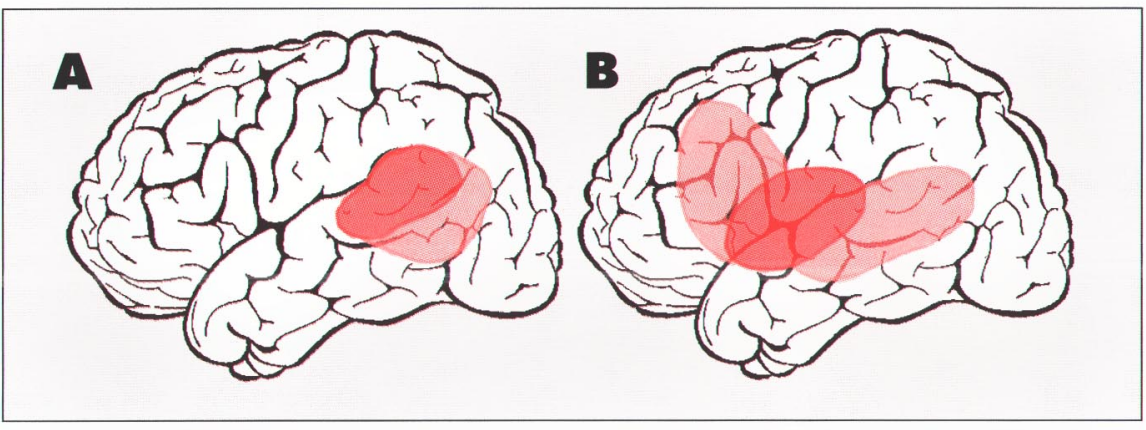

Figure 4. Schématisation des lésions provoquant une dyslexie de surface (A) et une dyslexie phonologique ou profonde (B). En réalité, l'étude des lésions responsables de dyslexie phonologique et de surface n'a pas permis de distinguer clairement les zones corticales sous-jacentes aux deux mécanismes postulés par les théories cognitivistes.

techniques depuis le début des années 1990 laisse entrevoir le rôle fondamental qu'elles pourraient jouer en complément des deux approches précédentes, l'enjeu étant clairement de tenter d'établir des liens entre des modes de pensée qui n'ont pas réussi, jusqu'à présent, à rendre compte de façon cohérente à la fois des processus mentaux et des mécanismes cérébraux qui les soustendent.

Les premiers travaux réalisés jusqu'ici dans le domaine de la lecture avec les méthodes d'imagerie fonctionnelle in vivo, ont principalement utilisé la TEP (tomographie par émission de positons), méthode isotopique fondée sur la mesure des modifications des débits sanguins régionaux* après l'injection intraveineuse d'un traceur radioactif (eau marquée à l'oxygène 15).

Les premières études réalisées à l'aide de cette méthode furent celles de l'équipe de Raichle (Saint-Louis, MI, USA) [23]. Ces auteurs ont étudié les modifications de débit cérébral selon un protocole de "soustraction hiérarchique » destiné à isoler les composants cognitifs liés à diverses étapes du traitement du mot écrit. Pour ce faire, ils utilisèrent un proto-

\footnotetext{
* Il faut préciser que les résultats obtenus en TEP sont sujets à caution en raison d'incertitudes méthodologiques inhérentes à cette méthode: nécessité de moyennage de plusieurs sujets pour obtenir un signal suffisant, résolution temporelle faible, et surtout nécessité de "soustraire" l'état cognitif observé d'un état de référence. Notre propos n'étant pas ici de discuter de ces questions méthodologiques, nous renvoyons le lecteur à des articles récents [19-22] où ces
}

cole hiérarchisé dans lequel ils présentaient passivement aux sujets quatre listes de stimulus visuels similaires d'un point de vue perceptif: des suites de pseudo-lettres, des suites de consonnes réalisant un pseudomot non prononçable (LTZMG), des suites de lettres réalisant un pseudomot prononçable (TWEAL) et enfin des vrais mots de la langue anglaise. Alors que les deux premiers types de stimulus provoquaient une activation bilatérale dans la région des aires visuelles primaires, les vrais mots et les pseudo-mots prononçables, par opposition aux suites non prononçables, entraînaient l'activation unilatérale d'une zone de cortex visuel associatif dite "extrastriée », sur la face interne du lobe occipital gauche (gyrus lingual). Enfin, seuls les vrais mots, à l'inverse des trois autres types de stimulus, activaient une zone préfrontale inférieure gauche à laquelle les auteurs attribuèrent une fonction sémantique. Le résultat important et inattendu de cette expérience était donc l'activation de la zone occipitale extrastriée gauche, activation que les auteurs interprétaient comme traduisant la mise en jeu, par des stimulus orthographiquement corrects ou seulement plausibles, de processus de traitement de la forme visuelle des mots. Un autre résultat important de ce travail fut de montrer que, contrairement aux modèles neurologiques classiques [12], dans aucune des conditions le mot lu n'activait ni le gyrus angulaire gauche (qui représente d'après les modèles classiques le siège du traitement de la forme visuelle des mots), ni les zones tem- porales externes gauches connues pour leur implication dans le traitement phonologique. En revanche, ces résultats sont en faveur des modèles cognitivistes de la lecture dans la mesure où ils démontrent que la lecture de mots peut se faire sans passage obligé par la phonologie.

Un autre groupe, celui de Frackowiak à Londres, a également étudié l'activation cérébrale en TEP lors de tâches de lecture. Ces auteurs [24] ont ainsi proposé à leurs sujets une liste de mots à lire à haute voix. Afin d'éliminer par soustraction les composantes articulatoire (liées à la prononciation à haute voix) et perceptive, ils ont mis au point une condition de référence comparable à la condition expérimentale mais utilisant des pseudo-lettres, à l'instar de Petersen et al. [23], en demandant au sujet de répéter un même mot à chaque présentation. La principale activation obtenue lors de cette soustraction fut localisée au niveau de la partie postérieure du gyrus temporal supérieur (aire 22), une zone de cortex auditif associatif, proche de la localisation théorique du gyrus angulaire, donc beaucoup plus compatible avec le modèle neurologique classique que le travail de Saint-Louis. En particulier, ce travail ne montrait aucune activation particulière dans la zone extrastriée gauche dont l'importance était suggérée par le travail de Petersen. Des travaux plus récents, utilisant une méthode isotopique plus sensible [25], ont démontré, lors de la présentation visuelle de mots, l'activation d'une vaste zone corticale bilatérale, avec des pics plus importants aux niveaux occipital et temporal supérieur et moyen, bilatéraux mais prédominant à gauche. Cette prédominance gauche est plus nette pour la lecture à haute voix que la lecture silencieuse, ce qui s'explique par une plus forte sollicitation de l'hémisphère gauche lorsqu'il y a articulation des mots.

Une cause de discordances entre ces différents travaux pourrait être que, jusqu'à présent, il était nécessaire d'effectuer le moyennage de plusieurs sujets différents (6 à 8 ) pour obtenir un signal suffisamment net. Les perfectionnements récents de la méthode permettent à présent d'explorer de manière satisfaisante 


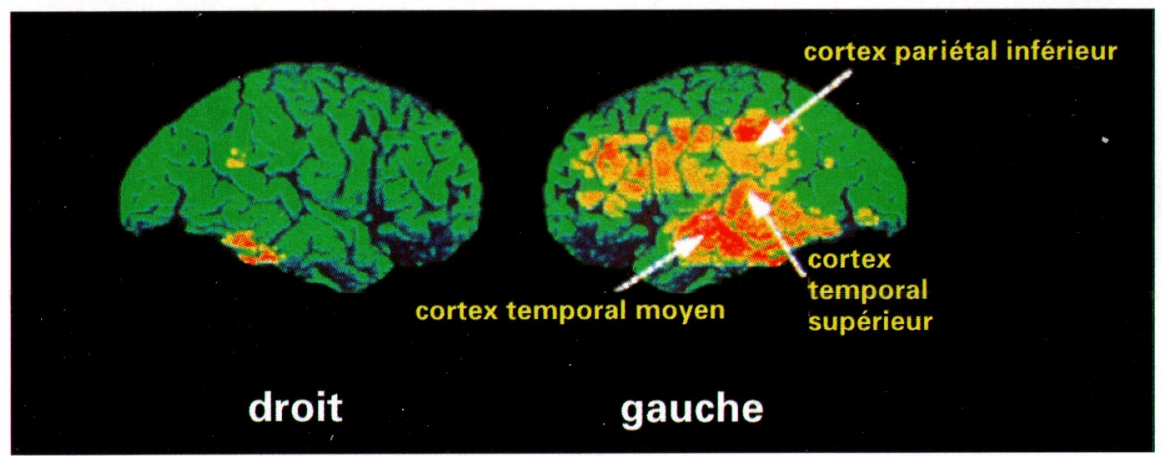

Figure 5. Cartographie fonctionnelle du cerveau par tomographie à positons au cours d'une tâche de lecture silencieuse de mots. Étude d'un sujet volontaire sain unique. Une vaste zone hémisphérique gauche est activée, incluant en particulier les aires temporales moyenne et postérieure et, à un moindre degré, du cortex extrastrié de manière bilatérale. On peut noter que les régions occipitales ne sont pas activées en raison de la nature visuelle de la tâche de référence (fixation d'un point). (Image MRC Cyclotron Unit, Pr. Frackowiak).

des cas uniques (figure 5) et devraient donc apporter un nouvel éclairage par la prise en compte des différences interindividuelles. Dans le même ordre d'idée, une autre technique d'imagerie fonctionnelle, celle utilisant la résonance magnétique (IRM fonctionnelle) devrait, dans les années à venir, contribuer à élargir le champ des connaissances dans le domaine.

\section{La dimension du développement: troubles de l'apprentissage et dyslexie de développement}

Comprendre les liens entre le cerveau et les fonctions cognitives n'est pas seulement faire "l'état des lieux» sur le cerveau adulte, c'est également essayer d'approcher les étapes qui, au cours de la maturation, ont amené à cet état, et les facteurs qui ont pu influencer cette maturation. C'est dans cette perspective que la neurologie cognitive se penche depuis peu sur les troubles spécifiques de l'apprentissage du langage écrit, ou dyslexie de développement, et leurs conséquences chez l'adulte. Cette anomalie du développement, qui touche environ $10 \%$ de la population d'enfants et d'adultes, est actuellement considérée comme la traduction d'un défaut de maturation, de cause encore inconnue mais probablement en dissociations dans leurs profils d'erreurs de lecture tout à fait similaires à celles observées chez l'adulte devenu alexique après une lésion cérébrale. On distingue ainsi des cas de dyslexie de surface et des cas de dyslexie profonde liées au développement, en fonction de la prédominance des erreurs de lecture de nonmots ou de mots irréguliers [26].

D'un point de vue cognitif, la dyslexie liée au développement est un terrain de choix pour comprendre les liens entre processus visuels et phonologiques dans la lecture. En effet, hormis les anomalies de la lecture et de l'orthographe, la grande majorité des dyslexiques présentent d'importantes difficultés dans des tâches purement phonologiques, comme segmenter un mot entendu en ses constituants sonores, ou encore juger si deux mots entendus riment ou non. Il a été prouvé que l'intensité de ces troubles, dits de "conscience phonologique», est proportionnelle à la sévérité de la dyslexie et on considère généralement qu'ils constituent la marque d'un trouble de la maturation, survenu dans la période prénatale, de cir-

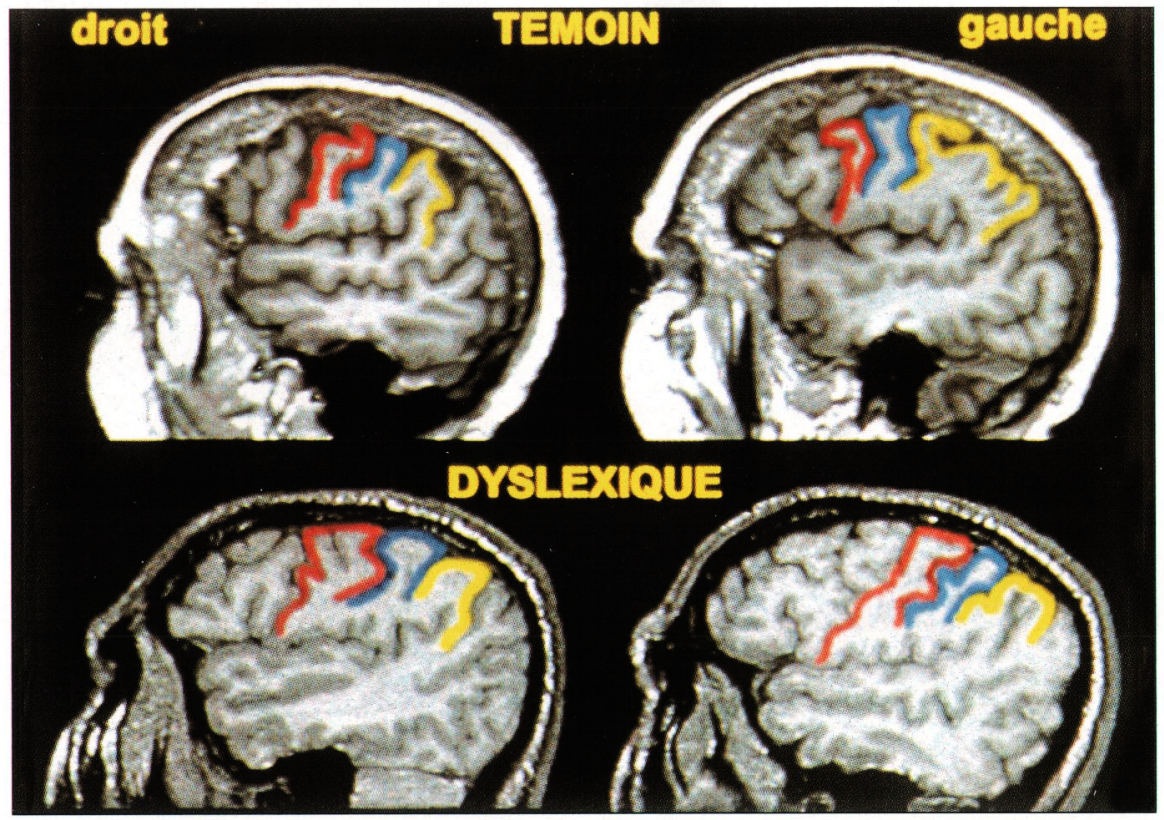

Figure 6. Asymétrie de l'opercule pariétal mise en évidence in vivo en imagerie par résonance magnétique. On retrouve, chez le sujet ayant souffert de dyslexie de développement, comparé aux témoins, une réduction de l'asymétrie entre les régions operculaires droite et gauche (région incluant le gyrus supra-marginal, en jaune sur la figure). Cet aspect est corrélé au degré de déficit phonologique: I'asymétrie est d'autant plus réduire que la performance du sujet dans des tâches phonologiques est plus faible. 
cuits cérébraux, principalement hémisphériques gauches, spécifiquement impliqués dans ces fonctions. En l'absence de ces mécanismes de traitement des formes sonores verbales, la correspondance sonore des graphèmes et des mots ne peut être apprise correctement, entravant l'apprentissage du langage écrit.

On ne connaît pas encore le substrat cérébral exact de ce trouble phonologique, mais diverses équipes se penchent actuellement sur la question à l'aide d'outils d'exploration divers, tels que les potentiels évoqués cognitifs, ou encore les méthodes d'imagerie fonctionnelle citées au paragraphe précédent. Toutefois, une approche strictement morphologique s'est récemment avérée capable de fournir des données précieuses en montrant, en particulier, de subtiles différences dans l'anatomie corticale entre sujets dyslexiques et non dyslexiques et, surtout, en démontrant un lien entre ces particularités morphologiques et les capacités phonologiques des sujets (figure 6). Il est probable que c'est la combinaison de ces différentes approches, clinique, expérimentale, cognitive, morphologique et fonctionnelle, et de la mise en parallèle de différents cadres théoriques, qui permettra, dans un proche avenir, de tirer tous les enseignements de ce modèle passionnant des relations cerveau/cognition que constituent la lecture et sa pathologie acquise ou liée au développement

\section{TIRÉS À PART}

M. Habib.

\section{Summary}

Brain mechanisms of reading: a model in cognitive neurology

The issue of brain mechanisms underlying the processes of reading has largely contributed, during the last century, to the development of models and new theoretical frameworks about brain-cognition relationships. Anatomo-clinical studies of alexia, a condition where patients become unable to read as a result of focal brain lesions, have been at the origin of the highly influential deconnectionist accounts to the classical neuropsychological syndromes. The cognitivist revolution, during the past 20 years, also started from dissociations observed in acquired reading impairments to provide neuropsychology with a new approach of the same syndromes. The current movement of cognitive neurology brings further elements of response to some hitherto unanswered questions, especially by the means of modern techniques of brain functional imaging, whose first results hold out the promise of bringing together the neuro-anatomical and cognitivist conceptions. Finally, considering the problem from a developmental perspective appears more and more as a necessary step toward understanding the structural bases of human cognition. Here again, it is the neurological approach of reading disabilities (developmental dyslexia) that has yielded the first convincing evidence of an anomalous neural organization as a basis of specific cognitive impairments. 